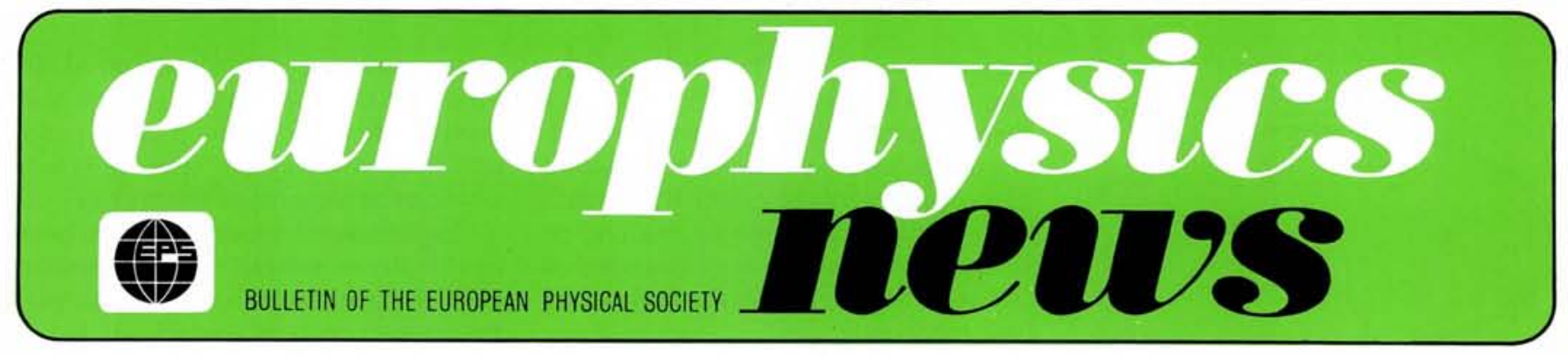

J.A.

Volume 19

Number 9

September 1988

\title{
Single-atom Oscillators
}

\section{Herbert Walther}

(Sektion Physik der Universität München und

Max-Planck-Institut für Quantenoptik, Garching/FRG)

Chairman of the EPS Quantum Optics Division

Modern methods of laser spectroscopy allow us to study single atoms or ions in an unperturbed environment. This has opened up interesting new experiments, among them the detailed study of radiation-atom coupling. In the following, two experiments of this type are reviewed: the single-atom maser and the resonance fluorescence of a single stored ion.

The simplest and most fundamental system for studying radiation-matter coupling is a single two-level atom interacting with a single mode of an electromagnetic field in a cavity. It received a great deal of attention shortly after the maser was invented, but at that time, the problem was of purely academic interest as the matrix elements describing the radiation-atom interaction are so small, the field of a single photon is not sufficient to lead to an atom-field evolution time shorter than other characteristic times of the system, such as the excited state lifetime, the time of flight of the atom through the cavity and the cavity mode damping time. It was therefore not possible to test experimentally the fundamental theories of radiation-matter interaction which predicted amongst other effects:

(a) a modification of the spontaneous emission rate of a single atom in a resonant cavity,

(b) oscillatory energy exchange between a single atom and the cavity mode, and

(c) the disappearance and quantum revival of optical (Rabi) nutation induced in a single atom by a resonant field.
The situation has changed drastically in the last few years with the introduction of frequency-tunable lasers that can excite large populations of highly excited atomic states, characterized by a high main quantum number $n$ of the valence electron. These states are generally called Rydberg states since their energy levels can be described by the simple Rydberg formula. Such excited atoms are very suitable for observing quantum effects in radiation-atom coupling for three reasons. First, the states are very strongly coupled to the radiation field (the induced transition rates between neighbouring levels scale as $n^{4}$ ); second, transitions are in the millimetre wave region, so that low-order mode cavities can be made large enough to allow rather long interaction times; finally, Rydberg states have relatively long lifetimes with respect to spontaneous decay (for reviews see Refs. 1 and 2).

The strong coupling of Rydberg states to radiation resonant with transitions to neighbouring levels can be understood in terms of the correspondence principle: with increasing $n$ the classical evolution frequency of the highly excited electron becomes identical with the transition frequency to the neighbouring level; the atom therefore corresponds to a large dipole oscillating with the resonance frequency. (The dipole moment is very large since the atomic radius scales with $n^{2}$ ).

\section{Spontaneous Emission Rate}

In order to understand the modification of the spontaneous emission rate in an external cavity, we have to remember that in quantum electrodynamics this rate is determined by the density of modes of the electromagnetic field at the atomic transition frequency $\omega_{0}$ which in turn depends on the square of the frequency. If the atom is not in free space, but in a resonant cavity, the continuum of modes is changed into a spectrum of discrete modes of which one may be in resonance with the atom. The spontaneous decay rate of the atom in the cavity $\gamma_{c}$ will then be enhanced in relation to that in free space $\gamma_{f}$ by a factor given by the ratio of the corresponding mode densities:

$$
\begin{aligned}
\gamma_{c} / \gamma_{f} & =\rho_{c}\left(\omega_{0}\right) / \rho_{f}\left(\omega_{0}\right) \\
& =2 \pi Q N_{c} \omega_{0}{ }^{3}=Q \lambda_{0}{ }^{3} / 4 \pi^{2} V_{c}
\end{aligned}
$$

where $V_{c}$ is the volume of the cavity and $Q$ is a quality factor of the cavity which

\section{Contents}

Single-atom Oscillators 105

Nobel Laureates Meet

Physics and Mathematics 109

Twenty Years On 110 EPS Under the Microscope 112 First Reactions of the

Executive Committee

Pellet Fuelling of Tokamaks 115

Physical Society of Japan 118

East-West Schools of Physics 


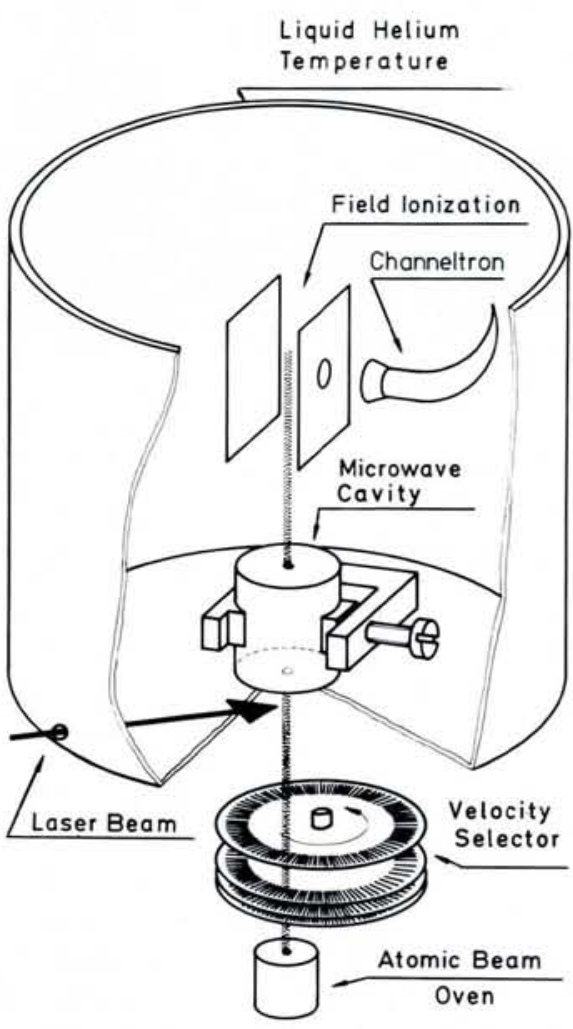

Fig. 1 - Scheme of the single-atom maser for measuring quantum collapse and revivals (see Ref. 8).

expresses the sharpness of the mode. For low-order cavities in the microwave region $V_{c} \cong \lambda_{0}{ }^{3}$ which means that the spontaneous emission rate is increased by roughly a factor of $Q$. However if the cavity is detuned, the decay rate will decrease. In this case, the atom cannot emit a photon, since the cavity is not able to accept it, and therefore the energy has to stay with the atom.

Recently, a number of experiments have been made with Rydberg atoms to demonstrate this enhancement and inhibition of spontaneous decay in external cavities or cavity-like structures (for the most recent experiment see Ref. 3 ).

More subtle effects due to the change of the mode density can also be expected: radiation corrections such as the Lamb shift and the anomalous magnetic dipole moment of the electron are modified with respect to the free space value [4], although changes are of the same order as present experimental accuracy. Roughly speaking, one can say that such effects are determined by a change of virtual transitions and not by real transitions as in the case of spontaneous decay.

In the following, attention is focussed on the one-atom maser in which the idealized case of a two-level atom interacting with a single mode of a radiation field is realized. The theory of this system was treated by Jaynes and Cummings [5] many years ago and we shall concentrate on the dynamics of the atom-field interaction predicted by this theory. Some of the features are explicitly a consequence of the quantum nature of the electromagnetic field: the statistical and discrete nature of the photon field leads to new dynamic characteristics such as collapse and revivals in the Rabi nutation.

The experimental setup of the oneatom maser is shown in Fig. 1. The atoms are excited with frequencydoubled light of a dye laser to the $63 p_{3 / 2}$ Rydberg level and are then injected into a superconducting cavity. On emerging they are monitored using field ionization which can be performed state-selectively by choosing the proper field strength. The cavity is tuned by squeezing it with piezo electric elements. The flux of atoms is very low so that the average number in the cavity at a time is usually much less than unity.

In Fig. 2 is shown a typical lowering of the number of atoms in the upper state as the tuning of the cavity moves through the resonance. This is for the 63 $p_{3 / 2}-61 d_{3 / 2}$ transition although in most of the experiments $[6,7]$ the transition $63 p_{3 / 2}-61 d_{5 / 2}$ with a frequency of $21.456 \mathrm{GHz}$ has been investigated.

\section{Dynamics of the Single Atom - Single Mode Operation}

With very low atomic-beam fluxes, the cavity of the single-atom maser contains essentially thermal photons only, whose number varies randomly obeying Bose-Einstein statistics. At high fluxes, the atoms deposit energy in the cavity and the maser reaches the threshold so that the number of photons stored in the cavity increases and their statistics changes.

For a coherent field the probability distribution is Poissonian which results in a dephasing of the Rabi oscillations, and therefore the envelope of $P_{e}(t)$, the probability of finding the atom in the upper maser level, collapses. After the collapse, $P_{\mathrm{e}}(t)$ starts oscillating again in a very complex way. Such changes recur periodically, the time interval being proportional to $n^{1 / 2}$. Both collapse and revival in the coherent state are pure quantum features without any classical counterpart [7].

Collapse and revival also occur in the case of a thermal Bose-Einstein field where the spread in the photon number is far larger than for a coherent state, and the collapse time is much shorter. In addition, revivals overlap completely and interfere, producing a very irregular time evolution. On the other hand, a classical thermal field represented by an exponential distribution of the intensity shows collapse, but no revival at all. From this it follows that revivals are pure quantum features of the thermal radiation field, whereas the collapse is less clear-cut as a quantum effect [7].

The above-mentioned effects have been demonstrated experimentally, using a Fizeau velocity selector to vary the interaction time (see Fig. 1). Fig. 3 shows a series of measurements obtained with the single-atom maser [7], where $P_{\mathrm{e}}(t)$ is plotted against interaction time for increasing atomic flux $N$. The strong variation of $P_{\mathrm{e}}(t)$ for interaction times between 50 and $80 \mu$ s disappears for larger $N$ and a revival shows up for $N$ $=3000 \mathrm{~s}^{-1}$ for interaction times larger than $140 \mu \mathrm{s}$. The average photon number in the cavity varies between 2.5 and 5 , about 2 photons being due to the black-body field in the cavity corresponding to a temperature of $2.5 \mathrm{~K}$.

\section{Distribution of Cavity Photons}

There are two approaches to the quantum theory of the one-atom maser. Filipowicz et al. [9] use a microscopic approach to describe the device while Lugiato et al. [10] use the standard macroscopic quantum laser theory and arrive at the same steady-state photon number distribution. The special features of the micromaser are not emphasized in standard laser theory because the broadening due to spontaneous decay obscurs the Rabi cycling of the atoms. When similar averages in the

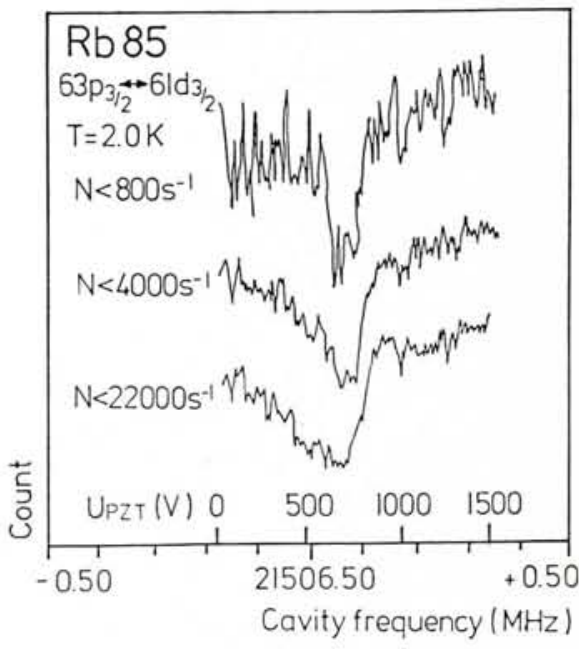

Fig. 2 - Maser resonance at a cavity temperature of $2 \mathrm{~K}$. The three recordings show the population in the upper state versus resonance frequency of the cavity. For the atomic flux of 800 atom/s there are on the average 1.5 photons in the cavity due to the blackbody radiation and 2 photons generated by the Rydberg atoms. At this flux there are only 0.06 atoms on the average in the cavity at any one time. At higher fluxes power broadening and an AC Stark shift of the resonances is observed (for details see Ref. 6). 

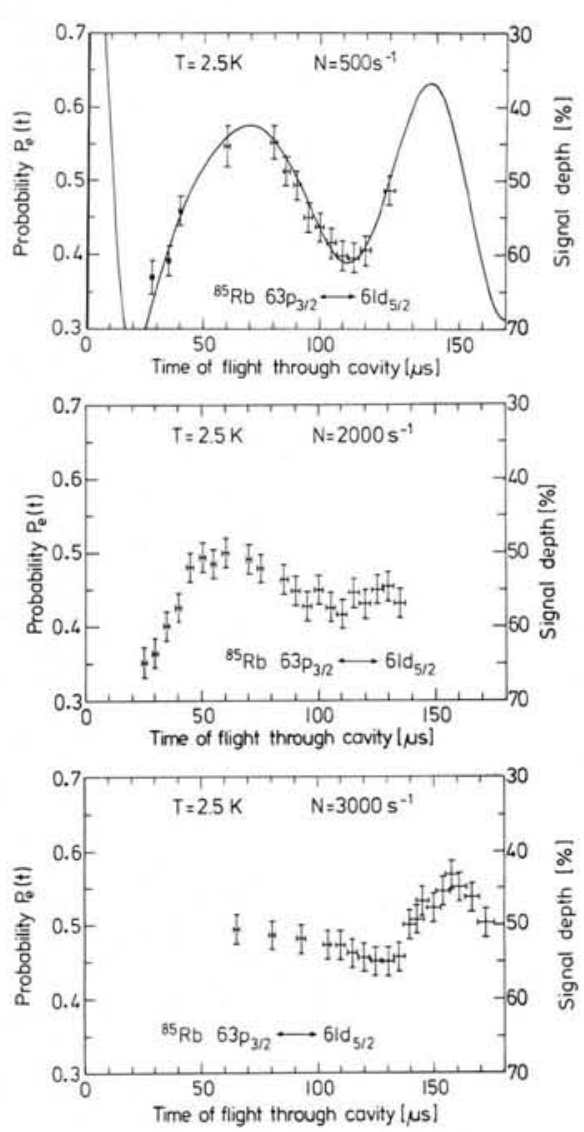

Fig. 3 - Quantum collapse and revival in the one-atom maser. Plotted is the probability $P_{e}(t)$ of finding the atom in the upper maser level for different fluxes $\mathrm{N}$ of the atomic beam (see Ref. 8).

microscopic theory associated with inhomogeneous broadening are performed, equivalent results are obtained.

Both theoretical approaches predict that the distribution of the photons in the maser cavity depends on the interaction time of the atoms in the cavity. It is mostly sub-Poissonian, and with a high $Q$ cavity, $Q \cong 5 \times 10^{10}$, the photon number reaches a steady value i.e. a Fock state [11].

To achieve such a state experimentally, two conditions have to be met. The first concerns the temperature; thermal photons have to be suppressed because they not only induce statistical decay but also result in a superposition of number states. The second is that losses of photons stored in the cavity can be neglected which means that the $Q$ value has to be higher than $10^{10}$. Both conditions can now be realized and the predicted distributions demonstrated.

New insight has been obtained into the statistical properties of ordinary masers and lasers where, in contrast to the single-atom maser, Poisson statistics is observed. The reason for this is that in the cavities of macro-masers and lasers there is much stronger damping than in the micromaser; in addition, atomic or molecular transitions usually show stronger damping than the Rydberg states. Furthermore, the selected velocity of the atoms used in connection with the collapse and revival measurements [8] in the micromaser leads to fixed interaction times in the cavity; this also helps to reduce the photon number fluctuations since the photon exchange between the atom and cavity field can be exactly controlled. The smallest fluctuations are achieved when the atoms leave the cavity again in the upper state. Of course, it is necessary that energy be deposited in the cavity in order to maintain maser oscillation, but the losses are very small with a high- $Q$ cavity and $P_{\mathrm{e}}$ for atoms leaving the cavity can be made very close to unity.

There are other interesting aspects of Rydberg masers which can only be briefly discussed here. Recently, a two-photon maser was realized by Brune et al. [12]. The two-photon transition was chosen such that there is an intermediate level nearly halfway between the upper and lower maser levels, thus enhancing the transition amplitude. In such a device new features not present in one-photon masers can be observed, e.g. delayed start-up time at threshold or multistable behaviour. Unlike the onephoton maser, which behaves at threshold similarly to a 2nd-order phase transition, the two-photon maser is analogous to a 1st-order phase transition. At this point we should also mention that it has been pointed out by Meystre and co-workers that the micromaser can be used to investigate aspects of chaos and problems of measurement theory [13].

\section{Resonance Fluorescence}

Another problem in radiation-matter interaction which has received a lot of interest is resonance fluorescence. Unlike the one-atom maser, here the atom decays under the influence of the vacuum fluctuations of free space and the emitted photons disappear and cannot be reabsorbed.

Most current work is concerned with theorically and experimentally determining the spectrum of the fluorescent light radiated by a two-level atom driven by an intense monochromatic field. This is the situation that gives rise to a dynamic Stark effect in which, for sufficiently strong fields, it is found that the spectrum of the scattered light splits into three peaks: a central peak, centred on the driving field frequency with a width $\gamma / 2(1 / \gamma$ being the Einstein A coefficient) and a height three times that of two symmetrically placed sidebands, each of width $3 \gamma / 4$ and displaced from the central peak by the Rabi frequency. In addition, there is a delta-function (coherent) contribution, also positioned at the driving frequency. In the limit of strong driving fields, the energy carried by this last contribution is negligible in relation to the three-peak contribution. This result was first predicted by Mollow and has now been confirmed experimentally (for a review see Refs. 14 and 15).

However, it is not only the spectrum of the fluorescent light that has come under investigation. Examination of the intensity correlation of the scattered field in the basic two-level atom has also attracted much attention since fluorescent light exhibits interesting statistical

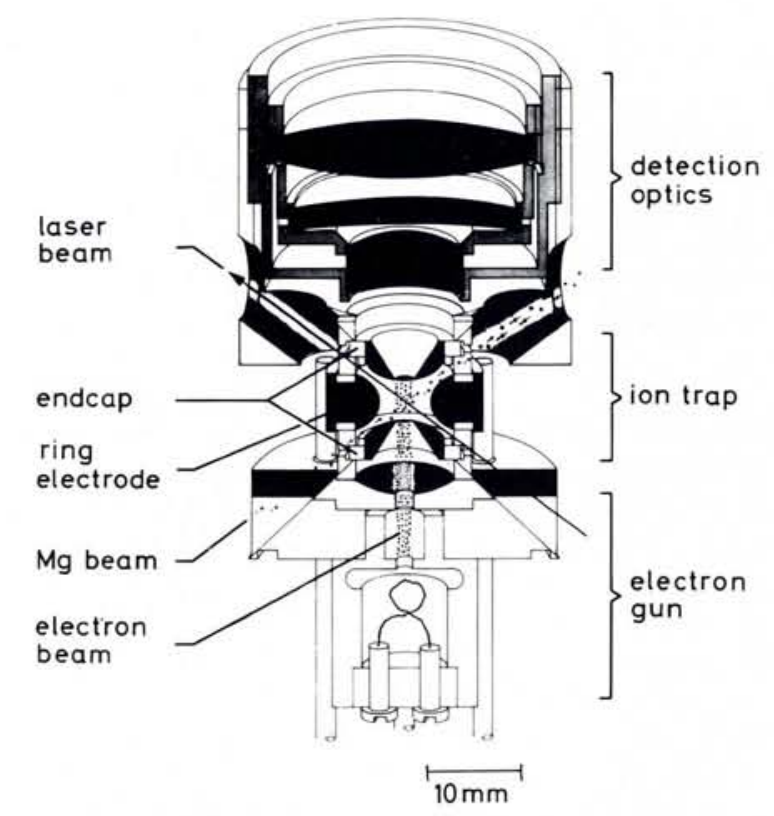

Fig. 4 - Scheme of the Paul trap used for the experiments (for details see Ref. 16). 
properties, especially when there is only a single atom at a time interacting with the laser beam. Under these conditions, the phenomenon of photon-antibunching can be observed [14, 15]. The single-atom condition cannot easily be fulfilled if experiments are made on neutral atoms, whereas the new techniques of laser spectroscopy of single ions in a radio-frequency trap are very suitable for this purpose, as has recently been demonstrated by Diedrich and Walther [16].

The Paul trap used in the experiment is shown in Fig. 4. Mounted inside a stainless-steel ultrahigh vacuum chamber, its ring diameter of $5 \mathrm{~mm}$ and its pole cap separation of $3.54 \mathrm{~mm}$ are much larger than for other single-ion radiofrequency traps. The single ion is closely confined in the centre of the trap by photon recoil cooling [9]. The large size of the trap affords a large solid angle for detecting the fluorescence radiation which is transmitted through a molybednum mesh covering a conical bore in the upper pole cap. The experiment has been performed with ${ }^{24} \mathrm{Mg}^{+}$ions produced by an atomic beam ionized in the centre of the trap by an electron beam. The resonance transition has the wavelength $\lambda=280 \mathrm{~nm}$.

To investigate the photon statistics, the second-order correlation function (intensity correlation) was measured in a Hanbury-Brown and Twiss Experiment. The intensity correlation is proportional to the probability of detecting a second photon at a time $\tau$ after the first. For thermal and non-coherent light this probability has a maximum for $\tau=0$ and decreases for larger $\tau$. The behaviour is called bunching. The intensity correlation for coherent light is independent of $\tau$. Quantized fields may show additional behaviour: the intensity correlation can have a minimum at $\tau=0$, a behaviour known as antibunching. Such a field is produced by the single stored ion in the following way: after a photon is emitted, the trapped ion returns to the ground state; before the next photon can be emitted, the ion has to be excited again. This happens through Rabi nutation in the external laser field. On the average, a time of half a Rabi period has to elapse until another photon can be observed. The probability of two photons being emitted in a short time interval is therefore zero.

The results for the intensity correlation of a single stored ion are shown in Fig. 5. Plotted is $g_{l}^{(2)}(\tau)$, which is defined by: $g_{l}^{(2)}(\tau)=\langle I(t) \|(t+\tau)\rangle \mid\langle I(t)\rangle^{2}$.

Owing to a time delay in one of the signal channels the intensity correlation $g_{i}^{(2)}(\tau)$ could also be measured for negative $\tau$. The laser intensity decreases from a to $d$ and therefore the average time interval in which a second photon follows a first increases.

The fluorescent light of a single stored ion has another interesting property: the fluctuations of the photon number recorded in a small time interval $\delta t$ is narrower than that expected for a Poisson distribution, i.e. the variance is smaller than the mean value of the photon number and we again find sub-Poissonian statistics. The reason is that the single ion can only emit one photon at a time and fluctuations only occur because of the finite detection probability. Antibunching and sub-Poissonian statistics are often associated, although they are distinct properties and need not necessarily be simultaneously observed, as is the case in the experiment described here. However, they are non-classical properties of the radiation field; a semiclassical treatment, where the electromagnetic field is not quantized, does not show this behaviour.

Another interesting phenomenon recently observed by Diedrich et al. [17] in a Paul trap concerned clouds of 2 to about 50 simultaneously stored $\mathrm{Mg}^{+}$ ions. Two phases of the ions could be clearly distinguished by their excitation spectra, one corresponding to a cloudlike state, in which the ions move randomly, and the other to a crystalline state, in which they are fixed in a regular array. The latter state is determined by the pseudopotential of the trap and the Coulomb repulsion of the ions. Such crystals are formed when laser cooling reduces the kinetic energy of the ions to a value which is much smaller than the potential energy of the ions in the trap.

\section{REFERENCES}

[1] Haroche S. and Raimond J.M. in Advances in Atomic and Molecular Physics, Vol. 20 , eds. D. Bates and B. Bederson (Academic Press) 1985, p. 347

[2] Gallas J.A.C., Leuchs G., Walther H. and Figger H., ibid. p. 413.

[3] The W., Anderson A., Hinds E.A., Meschede D., Moi L. and Haroche S., Phys. Rev. Lett. 58 (1987) 666.

[4] Barton G., Proc. Roy. Soc., A410 (1987) 147 and 175.

[5] Jaynes E.T. and Cummings F.W., Proc. IEEE 51 (1963) 89.

[6] Meschede D., Walther H. and Müller G., Phys. Rev. Lett. 54(1985) 551.

[7] Yoo H.I. and Eberly J.H., Phys. Rep. 118 (1985) 239 and Knight P.L. and Radmore P.M., Phys. Lett. 90A (1982) 342

[8] Rempe G., Walther H. and Klein N., Phys. Rev. Lett. 58 (1987) 353.

[9] Filipowicz P., Javanainen J., Meystre P., Phys. Rev. A34 (1986) 3077.

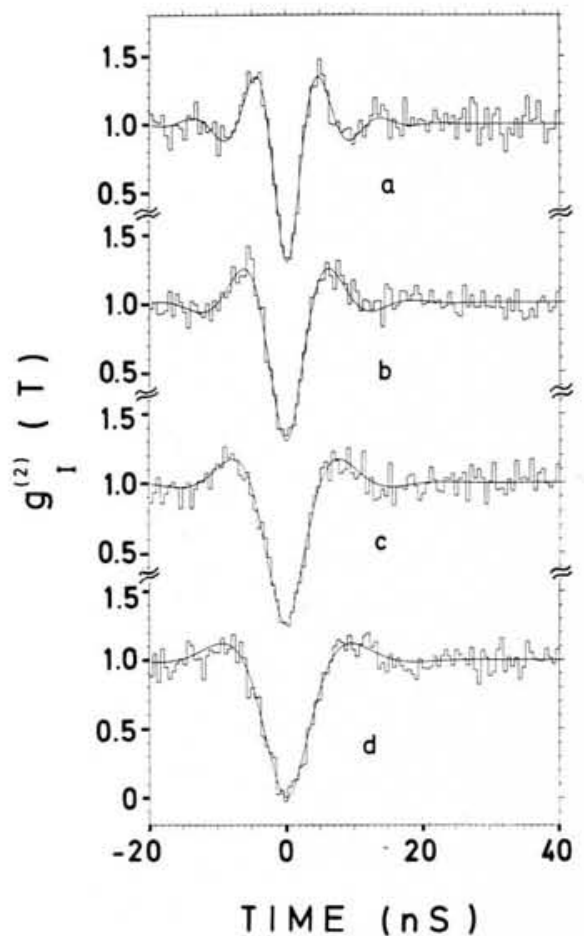

Fig. 5 - Results for the intensity correlation. Antibunching for a single ion for different laser intensities, increasing from bottom to top (see Ref. 16 for details).

The ion crystals in the trap represent a very neat model system and it is of considerable interest to investigate how the conditions depend on the stored ion number: certain ion configurations can be expected to be more stable than others and will therefore need less laser cooling when they are formed. Futhermore, the vibrational modes of the crystalline structure can also be investigated as well as the dynamics of the crystallization and evaporation process.

[10] Lugiato L.A., Scully M.O. and Walther $\mathrm{H}$. Phys. Rev. A 36 (1987) 740.

[11] Krause J., Scully M.O. and Walther H. Phys. Rev. A 36 (1987) 4547.

[12] Brune M., Raimond J.M., Goy P., Davidovich L. and Haroche S., Phys. Rev. Lett. 59 (1987) 1899.

[13] Meystre P., Opt. Lett. 12 (1987) 669 and Meystre P. and Wright E.M., Phys. Rev. A to be published.

[14] Cohen-Tannoudji C., in Frontiers in Laser-Spectroscopy, eds. R. Balian, S. Haroche and S. Liberman (North Holland) 1977.

[15] Cresser J.D., Häger J., Leuchs G., Rateike M. and Walther $\mathrm{H}$. in Dissipative Systems in Quantum Optics, ed. R. Bonifacio, Topics in Current Physics, Vol. 27 (Springer Verlag) 1982, 21.

[16] Diedrich F. and Walther H., Phys. Rev. Lett. 58 (1987) 203.

[17] Diedrich F., Peik E., Chen J.M., Quint W. and Walther H., Phys. Rev. Lett. 59 (1987) 2931. 